\title{
Recovering the Free-Field Acoustic Characteristics of a Vibrating Structure from Bounded Noisy Underwater Environments
}

\author{
Wei Lin ${ }^{1,2}$ and Sheng $\mathrm{Li}^{2, *}$ \\ 1 Qingdao Innovation and Development Center, Harbin Engineering University, Qingdao 266000, China; \\ linwei23@hrbeu.edu.cn \\ 2 State Key Laboratory of Structural Analysis for Industrial Equipment, School of Naval Architecture, \\ Dalian University of Technology, Dalian 116024, China \\ * Correspondence: shengli@dlut.edu.cn
}

Citation: Lin, W.; Li, S. Recovering

the Free-Field Acoustic

Characteristics of a Vibrating Structure from Bounded Noisy Underwater Environments. Sensors 2021, 21, 5521. https://doi.org/ $10.3390 / \mathrm{s} 2116552$

Academic Editor: Andrea Trucco

Received: 29 June 2021

Accepted: 12 August 2021

Published: 17 August 2021

Publisher's Note: MDPI stays neutral with regard to jurisdictional claims in published maps and institutional affiliations.

Copyright: (c) 2021 by the authors. Licensee MDPI, Basel, Switzerland. This article is an open access article distributed under the terms and conditions of the Creative Commons Attribution (CC BY) license (https:/ / creativecommons.org/licenses/by/ $4.0 /)$.

\begin{abstract}
The vibrational behavior of an underwater structure in the free field is different from that in bounded noisy environments because the fluid-structure interaction is strong in the water and the vibration of the structure caused by disturbing fields (the reflections by boundaries and the fields radiated by sources of disturbances) cannot be ignored. The conventional free field recovery (FFR) technique can only be used to eliminate disturbing fields without considering the difference in the vibrational behavior of the structure in the free field and the complex environment. To recover the free-field acoustic characteristics of a structure from bounded noisy underwater environments, a method combining the boundary element method (BEM) with the vibro-acoustic coupling method is presented. First, the pressures on the measurement surface are obtained. Second, the outgoing sound field and the rigid body scattered sound field are calculated by BEM. Then, the vibro-acoustic coupling method is employed to calculate the elastically radiated scattered sound field. Finally, the sound field radiated by the structure in the free field is recovered by subtracting the rigid body scattered sound field and the elastically radiated scattered sound field from the outgoing sound field. The effectiveness of the proposed method is validated by simulation results.
\end{abstract}

Keywords: free field recovery; free-field acoustic characteristics; bounded noisy underwater environment; boundary element method; vibro-acoustic coupling method

\section{Introduction}

It is important to characterize a target in an intrinsic way for target identification and control [1]. To acquire the intrinsic acoustic characteristics of the target, the measurement must be carried out in the free field. However, there is no ideal free field in nature or laboratory. Although the sound field radiated by a target in a large lake or anechoic tank can be approximated as a free sound field, the measurement is susceptible to bio-acoustic background noise and climate change in the large lake [2], and it is difficult to satisfy the free-field condition in the anechoic tank at low frequencies. To recover the free-field acoustic characteristics in the non-anechoic environment, the FFR techniques have been developed.

Pachner [3] initially showed a method separating the traveling and standing components on the surfaces of two spheres surrounding the source. The outgoing and incoming components of the sound field were separated based on spherical harmonics for two concentric spheres when the sound source is located inside the inner sphere [4]. Tsukernikov [5] combined the Helmholtz integral equation and spherical wave expansion method to realize the sound field calculation in a closed space. Williams [6] showed an approach to remove the disturbing fields based on spatial Fourier transform. This method has been widely applied to nearfield acoustic holography (NAH) $[7,8]$. However, this method can only be used for regular measurement surfaces and sound sources such as planes, cylinders, and 
spheres. The statistically optimized nearfield acoustic holography (SONAH) was developed to predict the pressure and velocity in complex environments $[9,10]$. However, this technique cannot accurately reconstruct the local sound field. To identify source velocities in complex environments, the inverse patch transfer functions method (iPTF) suitable for three-dimensional structures was proposed [11-14]. However, the problem of inversion of the ill-conditioned matrix needs to be solved when this method is used. The effects of both scattering (due to incident excitation) and radiation (due to interior noise sources) were removed throughout a "virtual" volumetric sonar array projected within the structure [15]. However, the capability to determine the range of near-field sources or scatterers needs to be improved. The supersonic intensity in a reverberant environment (SIRE) technique, which made use of an underwater vector sensor to obtain narrow-band sound power and directivity of a target in a reverberation environment was proposed [2]. However, the scattered sound field was not removed in this method. Langrenne et al. [16] presented a method based on BEM to recover the free sound field of a structure in a complex environment by considering the scattering effects on the machine. The equivalent source method (ESM)-based FFR technique combined with the NAH was used to reconstruct the sound field radiated by a target source from the measured mixed field [17-20]. An improved double-layer BEM was proposed to recover the half-space acoustic characteristics of a target source sitting on the boundaries in a bounded noisy environment by combining it with the image-source method [21]. Wu et al. [22] presented a BEM-based NAH in conjunction with the FFR technique to reconstruct the free sound field in a non-anechoic environment. However, the interaction between the structure and the fluid medium is not considered in these four methods. Although Sternini et al. [23] presented a method taking into account the vibrational response of an elastic object caused by incident wave, this method was used to calculate the bistatic scattered and needed to solve the problem of singular matrix inversion.

The conventional FFR technique does not take care of the difference between the vibrational behaviors of the structure in the free field and in the bounded noisy underwater environment. A method combining the BEM with the vibro-acoustic coupling method is proposed to recover the free-field acoustic characteristics in a bounded noisy underwater environment by considering the fluid-structure interaction. The goal of this article is to recover the free-field acoustic characteristics of the vibrating structure rather than to reconstruct the surface vibration of the structure. Thus, the NAH is not required. The theory of the proposed method is described in detail in Section 2. Section 3 validates the effectiveness of the proposed method through numerical simulations. Conclusions and suggestions for future research efforts are given in Section 4.

\section{Theory}

Figure 1 shows a structure vibrating in the free field and in a bounded noisy environment. As shown in Figure 1, the vibration of the structure is only caused by the force in the free field. However, the structural vibration in the bounded noisy environment is caused not only by the force but also by the disturbing fields (the reflections by boundaries and the fields radiated by sources of disturbances) [24]. For clarity, the vibrational behavior of the structure in the free field is defined as $V_{1}$ and the vibrational behavior of the structure in the bounded noisy environment is defined as $V_{2}$ in this paper. The elastic vibration of the structure caused by disturbing fields might be ignored in the air because the interaction between the structure and the air is weak. In this case, $V_{1}$ is the same as $V_{2}$. However, the elastic vibration of the structure caused by disturbing fields is not ignorable in the water due to the strong interaction between the structure and the water. This means that $V_{1}$ is different from $V_{2}$ in the water, and the sound field radiated by the structure in the free field is different from that in the bounded noisy environment. Thus, to recover the free-field acoustic characteristics radiated by the structure in the bounded noisy underwater environment, the vibration of the structure caused by disturbing fields must be considered, especially when the structure is a kind of thin-shell structure. 


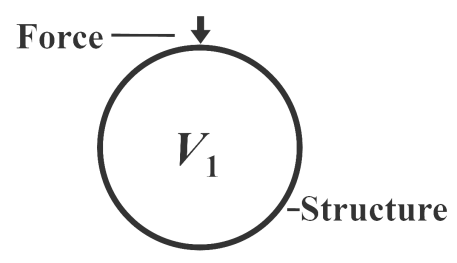

(a) In the free field

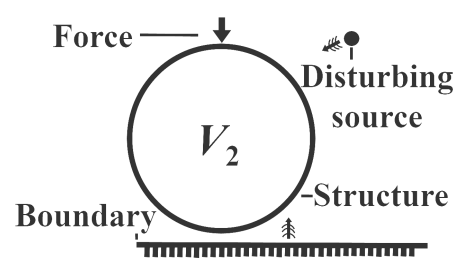

(b) In a bounded noisy environment

Figure 1. The vibrational behavior of the structure.

\subsection{Sound Field Separation}

For a vibrating structure in a complex underwater environment, as shown in Figure 2, $\Gamma_{1}$ is the surface of structure $S_{0} ; \Gamma_{2}$ represents a boundary of the sound field; $S$ is the measurement surface; the space between $\Gamma_{1}$ and $S$ is $A_{1}$, and the other space is $A_{2}$; $Q(r)$ represents the source of disturbance; and $n_{\Gamma_{1}}, n_{\Gamma_{2}}$, and $n_{S}$ are the normals to $\Gamma_{1}$, $\Gamma_{2}$, and $S$, respectively.

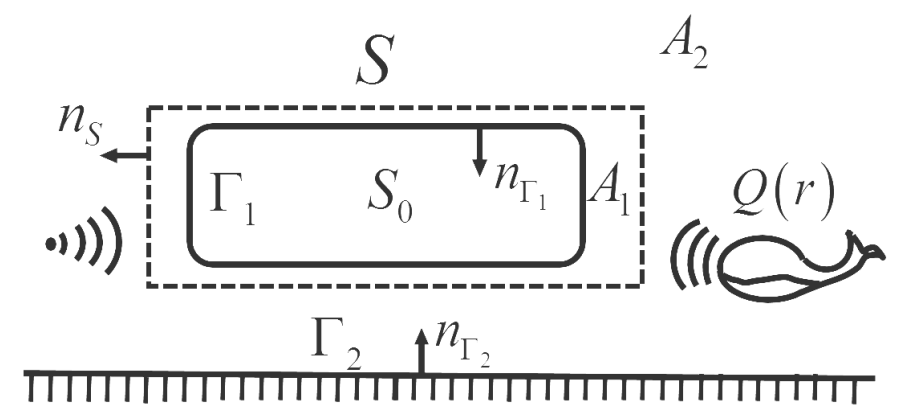

Figure 2. Geometry of a vibrating structure in a complex underwater environment.

The sound pressure $p(r)$ in a sound field is composed of incoming sound pressure $p^{i}(r)$ and outgoing sound pressure $p^{o}(r)$. It can be written as follows [16]:

$$
p(r)=p^{i}(r)+p^{o}(r)=p^{i}(r)+p^{f}(r)+p^{s}(r),
$$

It should be noted that $p^{i}(r)$ is composed of the sound pressure radiated by all sources of disturbances and reflected by the boundaries and that $p^{o}(r)$ consists of the free sound pressure $p^{f}(r)$ and the scattered sound pressure $p^{s}(r)$.

The scattered field is the superposition of an elastic contribution and a rigid contribution from the structure when sound waves are incident on the structure [23]. Thus, the scattered sound pressure $p^{s}(r)$ can be written as

$$
p^{s}(r)=p^{s r}(r)+p^{s e}(r),
$$

where $p^{s r}$ is the pressure scattered from an infinitely rigid body (rigid body scattered sound pressure) and $p^{s e}$ is the scattered component due to the elasticity of the structure (elastically radiated sound pressure) [23]. In the double-layer BEM [16], $p^{\text {se }}$ can be ignored due to industrial sources have much lower admittances than air. However, $p^{s e}$ is not ignorable in the water because of the strong interaction between the structure and the water.

When the field points in the $A_{1}$, this is an interior problem. The outgoing sound pressure $p^{o}(r)$ can be calculated from [16]

$$
\begin{aligned}
& p^{o}(r)-\int_{S}\left[p\left(s^{\prime}\right) \partial_{n} G\left(r, s^{\prime}\right)+i \rho \omega v_{n}\left(s^{\prime}\right) G\left(r, s^{\prime}\right)\right] d S \\
& = \begin{cases}p(r) & \text { for } \quad r \in A_{1} \\
c(r) p(r) & \text { for } \quad r \in S \\
0 & \text { for } \quad r \in A_{2},\end{cases}
\end{aligned}
$$


where $i, \rho, \omega$, and $G\left(r, s^{\prime}\right)$ are the imaginary unit, the fluid density, the circular frequency, and the three-dimensional free-space Green's function for Helmholtz equation, respectively. $c(r)$ is solid angle coefficient and is given by [25]

$$
c(r)=-\int_{S} \frac{\partial}{\partial n}\left(\frac{1}{4 \pi r}\right) d S,
$$

The sound pressure $p\left(s^{\prime}\right)$ and normal velocity $v_{n}\left(s^{\prime}\right)$ on the measurement surface $S$ can be calculated from the sound pressures on two parallel closed surfaces surrounding the structure [16]. Then, the $p^{o}\left(s^{\prime}\right)$ on the $S$ can be obtained from the Equation (3).

When the $p^{o}\left(s^{\prime}\right)$ is known, only $p^{s}\left(s^{\prime}\right)$ needs to be calculated to obtain $p^{f}\left(s^{\prime}\right)$. To calculate the scattered field, the incident field on the $\Gamma_{1}$ is necessary. When the field points in the $A_{2}$, this is an exterior problem. For the exterior problem, the incoming sound pressure on the $\Gamma_{1}$ can be solved by [16]

$$
\begin{aligned}
& p^{i}(r)+\int_{S}\left[p\left(s^{\prime}\right) \partial_{n} G\left(r, s^{\prime}\right)+i \rho \omega v_{n}\left(s^{\prime}\right) G\left(r, s^{\prime}\right)\right] d S \\
& =\left\{\begin{array}{lll}
0 & \text { for } & r \in A_{1} \\
\left(1-C^{0}(p)\right) p(r) & \text { for } & r \in S \\
p(r) & \text { for } & r \in A_{2} .
\end{array}\right.
\end{aligned}
$$

\subsection{Subtraction of the Scattered Field}

The Helmholtz integral equation for a scattered problem can be expressed as [25]

$$
\left(1-c^{\prime}(s)\right) p^{\prime}(s)=p^{i}(s)-\int_{\Gamma_{1}}\left[p^{\prime}(s) \partial_{n} G(r, s)+i \rho \omega v_{n}^{\prime}(s) G(r, s)\right] d S, \quad r \in A_{1}, A_{2},
$$

where $p^{\prime}(s)$ and $v_{n}^{\prime}(s)$ are respectively correspondent with the sound pressure and normal velocity on the $\Gamma_{1}$ at $s$ and where $c^{\prime}(s)$ is coefficient on the $\Gamma_{1}$.

According to the definition of the rigid body scattered sound pressure, $v_{n}$ equals zero. Then, the Equation (6) can be simplified as [16]

$$
\left(1-c^{\prime}(s)\right) p^{b}(s)=p^{i}(s)-\int_{\Gamma_{1}}\left[p^{b}(s) \partial_{n} G(r, s)\right] d S, \quad r \in \Gamma_{1},
$$

where $p^{b}$ is the blocked pressure, which is the sum of $p^{s r}$ and $p^{i}$ [23]. When $p^{i}$ is known, $p^{b}$ can be calculated by Equation (7). Furthermore, $p^{s r}$ on the $S$ radiated from $\Gamma_{1}$ can be computed by

$$
p^{s r}(r)=-\int_{\Gamma_{1}}\left[p^{b}(s) \partial_{n} G(r, s)\right] d S, \quad r \in S,
$$

The remaining problem is to calculate $p^{s e}$ since $p^{s r}$ is obtained. As mentioned before, $p^{s e}$ is radiated from elastic vibration of the structure caused by the incoming field. It is found that the elastic term $p^{\text {se }}$ is the elastically radiated component of the pressure field related to the elastic surface velocity $v_{n}^{s e}$, and together, they represent a radiation problem only, with no incident field involved [23]. In other words, $p^{\text {se }}$ and $v_{n}^{\text {se }}$ can be expressed as

$$
\left(1-c^{\prime}(s)\right) p^{s e}(s)=-\int_{\Gamma_{1}}\left[p^{s e}(s) \partial_{n} G(r, s)+i \rho \omega v_{n}^{s e}(s) G(r, s)\right] d S .
$$

When $v_{n}^{\text {se }}$ is given, $p^{\text {se }}$ can be acquired. Then, the problem is converted to calculate $v_{n}^{\text {se }}$. In order to compute $v_{n}^{s e}$, the fluid-structure coupling problem must be solved. The BEM has advantages in calculating the far-field sound pressure and can be used for fluid modeling [26]. In addition, the BEM can be coupled with the finite element method (FEM) to solve the fluid-structure coupling problem [27]. The coupled method is the vibro-acoustic coupling method. Considering that the indirect boundary element method (IBEM) can be used to calculate the internal and external sound fields at the same time, IBEM is coupled 
with FEM. First, the structure is discretized by FEM, and the corresponding finite element equation is [27]

$$
\left[K_{s}+i \omega C_{s}-\omega^{2} M_{s}\right][u]=\left[F_{s}\right],
$$

where $u$ is the displacement of structural node; $M_{s}, C_{s}$, and $K_{s}$ are the mass matrix, the damping matrix, and the stiffness matrix of the structure, respectively; $F_{s}$ is the mechanical load acting on the structure; and the time component is $e^{i \omega t}$, where $t$ is the time.

Then, the displacement and the sound field of the structure are solved simultaneously considering the continuity of the velocity at the coupling boundary. Ignoring the damping effect and satisfying the single layer potential, the Equation (10) coupled with IBEM can be expressed as [27]

$$
\left[\begin{array}{cc}
K_{s}+i \omega C_{s}-\omega^{2} M_{s} & L_{C} \\
L_{C}^{T} & \frac{D(\omega)}{\rho \omega^{2}}
\end{array}\right]\left[\begin{array}{l}
u \\
\mu
\end{array}\right]=\left[\begin{array}{l}
F_{s} \\
F_{a}
\end{array}\right]
$$

where $\mu$ is the double-layer potential, that is, the sound pressure difference on the structure surface; $L_{C}$ is the vibro-acoustic coupling matrix; the superscript letter $T$ stands for the transpose of matrix; $D(\omega)$ is the IBEM influence matrix; and $F_{a}$ is the acoustic load.

The mechanical load is equal zero when solving the scattered problem of the incident field on the elastic body. Additionally, it is noted that the acoustic load is approximated as $p^{b}(r)$ rather than $p^{i}(r)$. Equation (11) can be solved when the properties of structure are known before the sound field is recovered. Then, the surface velocity $v_{n}^{\text {se }}$ can be obtained from $u$. The $p^{s e}(r)$ on the $S$ can be calculated by the Helmholtz integral equation:

$$
\begin{aligned}
& -\int_{\Gamma_{1}}\left[p^{s e}(s) \partial_{n} G(r, s)+i \rho \omega v_{n}^{s e}(s) G(r, s)\right] d S \\
& =\left\{\begin{array}{ll}
p^{s e}(r) & , \quad r \in A_{1}, A_{2} \\
\left(1-c^{\prime}(s)\right) p^{s e}(s), & s \in \Gamma_{1}
\end{array} .\right.
\end{aligned}
$$

Using pressure fields $p^{o}(r), p^{\text {sr }}(r)$, and $p^{\text {se }}(r)$ computed from Equations (3), (8), and (12), respectively, the $p^{f}(r)$ is calculated from Equation (1):

$$
p^{f}(r)=p^{o}(r)-p^{s r}(r)-p^{s e}(r)=p_{V_{2}}^{f}(r)-p^{s e}(r), \quad r \in S
$$

where $p_{V_{2}}^{f}(r)$ is the sound field radiated by the structure for which the vibrational behavior is $V_{2}$, and it is clear that $p_{V_{2}}^{f}(r)$ is the sound field recovered by the double-layer BEM.

\subsection{Discretization}

To calculate the integral equations, the sufaces are discretized into elements. Then, the outgoing and incoming sound fields can be rewritten as

$$
\begin{gathered}
P_{S}^{o}=\left[C_{S}-H_{S}^{S}\right] P_{S}-i \rho \omega\left[G_{S}^{S}\right] V_{S}, \\
P_{\Gamma_{1}}^{i}=-\left[H_{S}^{\Gamma_{1}}\right] P_{S}-i \rho \omega\left[G_{S}^{\Gamma_{1}}\right] V_{S},
\end{gathered}
$$

where $C, H$, and $G$ are the solid angle coefficient matrix, the sound pressure, and the particle velocity transfer matrices, respectively. The subscript letter and the superscript letter stands for the integration surface and the target surface, respectively. Subsequently, the $P^{b}$ on the $\Gamma_{1}$ can be obtained from Equation (7):

$$
P_{\Gamma_{1}}^{b}=\left[I-C_{\Gamma_{1}}^{\prime}+H_{\Gamma_{1}}^{\Gamma_{1}}\right]^{-1} P_{\Gamma_{1}}^{i}
$$

where $I$ is the identity matrix and $C^{\prime}$ is the coefficient matrix. 
The combined Helmholtz integral equation formulation (CHIEF) method is used to overcome the non-uniqueness difficulty $[28,29]$. According to Equation (8), the sound pressure scattered from an infinitely rigid body on the $S$ can be given by

$$
P_{S}^{s g}=-\left[H_{\Gamma_{1}}^{S}\right] P_{\Gamma_{1}}^{b} .
$$

In order to calculate the response of the structure caused by the incoming field, the mechanical load is zero and the acoustic load is $p^{b}$. The properties including mass, the damping, and the stiffness of structure have to be known in advance. Then, the Equation (11) can be rewritten as

$$
\left[\begin{array}{cc}
K_{s}+i \omega C_{s}-\omega^{2} M_{s} & L_{C} \\
L_{C}^{T} & \frac{D(\omega)}{\rho \omega^{2}}
\end{array}\right]\left[\begin{array}{c}
U \\
\Phi
\end{array}\right]=\left[\begin{array}{c}
0 \\
P_{\Gamma_{1}}^{b}
\end{array}\right] .
$$

where $U$ is the displacement vector and $\Phi$ is the vector of the double-layer potential.

When the Equation (18) is solved, the normal velocity of structural surface $V_{\Gamma_{1}}^{s e}$ can be achieved by

$$
V_{\Gamma_{1}}^{s e}=[G][U],
$$

where $G$ is the transformation matrix, the function of which is to convert the displacement vector $U$ of the structural node into the normal velocity of the structure surface $V_{\Gamma_{1}}^{s e}$. Furthermore, the $P_{\Gamma_{1}}^{s e}$ can be achieved by

$$
P_{\Gamma_{1}}^{s e}=-i \rho \omega\left[I-C_{\Gamma_{1}}^{\prime}+H_{\Gamma_{1}}^{\Gamma_{1}}\right]^{-1}\left[G_{\Gamma_{1}}^{\Gamma_{1}}\right] V_{\Gamma_{1}}^{s e},
$$

Then, the $P_{S}^{s e}$ on the $S$ can be calculated from Equation (12)

$$
P_{S}^{s e}=-\left[H_{\Gamma_{1}}^{S}\right] P_{\Gamma_{1}}^{s e}-i \rho \omega\left[G_{\Gamma_{1}}^{S}\right] V_{\Gamma_{1}}^{s e}
$$

Finally, the free sound field recovered by the proposed method in the bounded noisy underwater environment is obtained using

$$
P_{S}^{f}=P_{S}^{o}-P_{S}^{s r}-P_{S}^{s e}
$$

\section{Numerical Simulations}

\subsection{In a Bounded Noisy Air Environment}

The numerical model is shown in Figure 3. The structure is a $1 \times 1 \times 1 \mathrm{~m}^{3}$ cubic shell and the side length $a=1$. The properties of the shell are shown in Table 1. The coordinate origin is at the centre of the shell. The structure is excited by a point force with amplitude $10 \mathrm{~N}$ and located at $(0 \mathrm{~m}, 0 \mathrm{~m},-0.5 \mathrm{~m})$, which is the centre of the upper surface. The upper surface of the structure is simply supported. The fluid medium is air. The sound speed in the air and the fluid density are $340 \mathrm{~m} / \mathrm{s}$ and $1.225 \mathrm{~kg} / \mathrm{m}^{3}$, respectively. In the air, the reference sound pressure is $2 \times 10^{-5} \mathrm{~Pa}$, and the reference sound power is $1 \times 10^{-12} \mathrm{~W}$. There is an infinite rigid plane at $z=1 \mathrm{~m}$ to simulate the rigid boundary such as the ground. A monopole located at $(0.75 \mathrm{~m}, 0.75 \mathrm{~m}, 0.75 \mathrm{~m})$ is used as a source of disturbance to influence the sound field. At $100 \mathrm{~Hz}$, the strength of the monopole is $-0.016 i \mathrm{~m}^{3} / \mathrm{s}$.

As the structure is a cubic shell, the strength of the fluid-structure coupling can be judged by the characteristic quantity $\lambda$ [30]:

$$
\lambda=\frac{\rho c}{\rho_{s} h \omega},
$$

where $c, h$, and $\rho_{s}$ are the sound speed in the fluid, the thickness of the plate, and the density of the plate, respectively. When $\lambda<1$, the coupling between the structure and the fluid is very weak; when $\lambda>1$, the coupling between the structure and the fluid is strong. 
When the cubic shell vibrates in the air, $\lambda$ is less than 0.1 in the studied frequency range. Thus, the coupling between the cubic shell and the air is very weak.

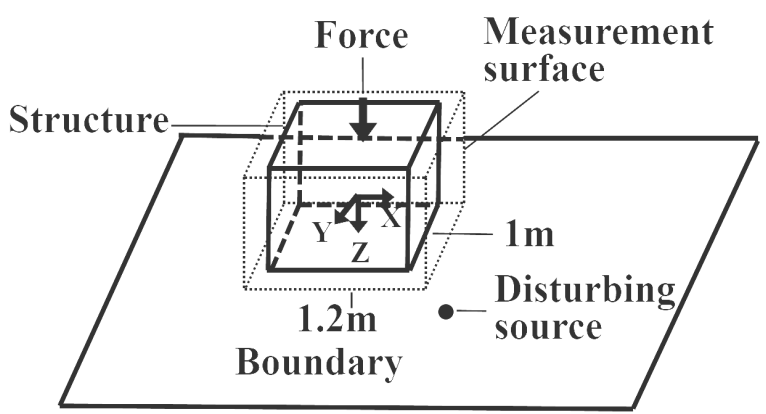

Figure 3. Geometry of the numerical model.

Table 1. The property of shell.

\begin{tabular}{ccccc}
\hline Thickness & Young Modulus & Poisson's Ratio & Density & Damping \\
\hline $0.005 \mathrm{~m}$ & $7 \times 10^{10} \mathrm{~N} / \mathrm{m}^{2}$ & 0.346 & $2710 \mathrm{~kg} / \mathrm{m}^{3}$ & 0 \\
\hline
\end{tabular}

The dimensions of the measurement surface $S$ are $1.2 \times 1.2 \times 1.2 \mathrm{~m}^{3}$. The distance between the two simulated layers located on each side of $S$ is $0.06 \mathrm{~m}$, which satisfies the convergence condition [20]. The measurement surface is discretized by 3456 linear quadrilateral elements with 3458 nodes. The structure is discretized by 2400 linear quadrilateral shell elements with 2402 nodes. The maximum frequency allowed by the mesh is $1133 \mathrm{~Hz}$, which corresponds to $k a=21$ ( $k$ is the wavenumber) based on the well-known $1 / 6$ criterion in the air. The maximum frequency allowed by the mesh is $5000 \mathrm{~Hz}$, which corresponds to $k a=21$ based on the $1 / 6$ criterion in underwater environments. It should be noted that, in the following numerical simulation, the sound pressure on the measurement surface is obtained from computation, not from real experimental testing.

First, the sound pressures on the measurement surface are calculated by the vibroacoustic coupling method in the free field when the structure is excited by the point force. The sound field in this case is defined as the $V_{1}$ free sound field. Second, the sound pressures on the two simulated layers are calculated by the vibro-acoustic coupling method when the structure is excited by the force in the bounded noisy environment. In this case, the directly obtained sound field is defined as the total sound field. Furthermore, the normal velocities on the surface of the structure that vibrate in the bounded noisy environment are taken as a boundary condition to compute sound field in the free field. Then, the calculated sound field is defined as the $V_{2}$ free sound field. Finally, the sound field recovered by the double-layer BEM and the sound field recovered by the proposed method are defined as the conventional recovered sound field labeled as c_recovered sound field and the new recovered sound field labeled as n_recovered sound field, respectively.

The power estimator is defined as [16]:

$$
I_{e}=\int_{S} \frac{\left|p^{e}(s)\right|^{2}}{\rho c} d S
$$

where $p^{e}(s)$ is sound pressure in the sound field. When calculating the power estimator in $V_{1}$ free sound field, $p^{e}(s)$ is equal to $p^{f}(s)$.

The mean quadratic error is used to compare the differences of sound fields. It is defined as [16]

$$
E=\sqrt{\frac{\int_{S}\left|p^{e}(s)-p^{F}(s)\right|^{2} d S}{\int_{S}\left|p^{F}(s)\right|^{2} d S}}
$$


where $p^{F}(s)$ is equal to $p^{f}(s)$, when calculating the mean quadratic error of sound field to $V_{1}$ free sound field. $p^{F}(s)$ is equal to $p_{V_{2}}^{f}$ when calculating the mean quadratic error of sound field to $V_{2}$ free sound field.

The power estimator levels directly obtained and recovered are shown in Figure 4. The power estimator level in the total sound field is very different from the power estimator level in the $V_{1}$ free sound field. The differences are more than $7 \mathrm{~dB}$ at most frequencies. This means that boundaries and sources of disturbances have a great influence on the acquisition of the free-field acoustic characteristics of the vibrating structure. However, the power estimator levels in the $V_{1}$ free sound field, in the $V_{2}$ free sound field, and in the conventional recovered sound field are the same, and the differences are less than $1 \mathrm{~dB}$.

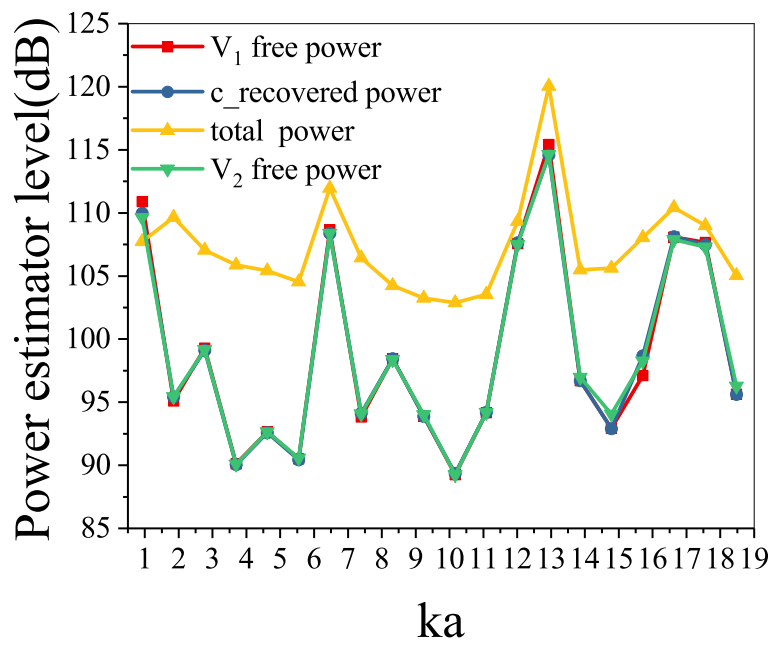

Figure 4. Sound power level on the measurement surface.

Figure 5 shows the contour map of sound pressure in the $V_{1}$ free sound field, the total sound field, the conventional recovered sound field, and the $V_{2}$ free sound field on the $S$. The sound pressure distribution of the $V_{1}$ free sound field is different from that of the total sound field. However, the sound pressure distributions of the $V_{1}$ free sound field, the $V_{2}$ free sound field, and the conventional recovered sound field are almost the same.

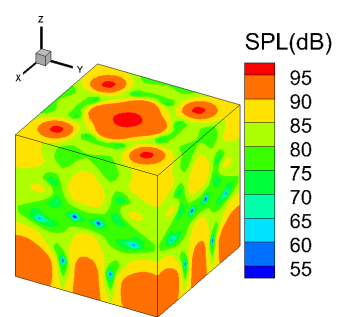

(a) $V_{1}$ free sound field

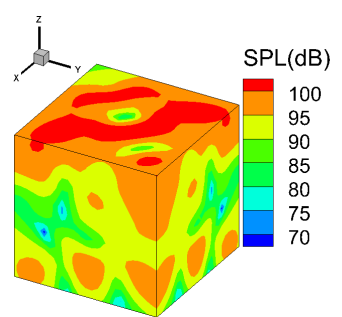

(b) total sound field

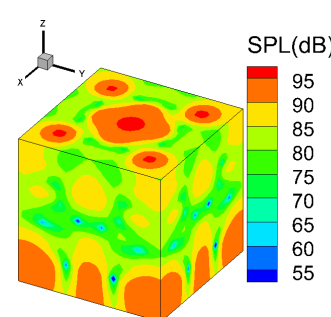

(c) c_recovered sound field

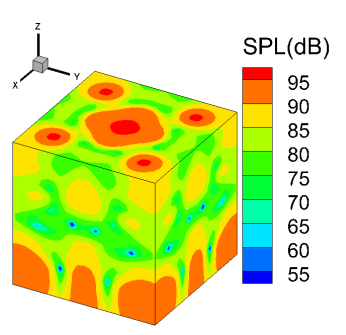

(d) $V_{2}$ free sound field

Figure 5. Contour map of sound pressure level (SPL) on the measurement surface at $450 \mathrm{~Hz}(\mathrm{ka}=8.3)$. 
The mean quadratic errors of the conventional recovered sound field, and the total sound field to the $V_{1}$ free sound field and the $V_{2}$ free sound field are shown in Figure 6. It shows that, at most frequencies, the mean quadratic errors of the total sound field to the $V_{1}$ free sound field and the $V_{2}$ free sound field are over $100 \%$. Meanwhile, the mean quadratic errors of the conventional recovered sound field to the $V_{1}$ free sound field and the $V_{2}$ free sound field are mostly below $10 \%$.

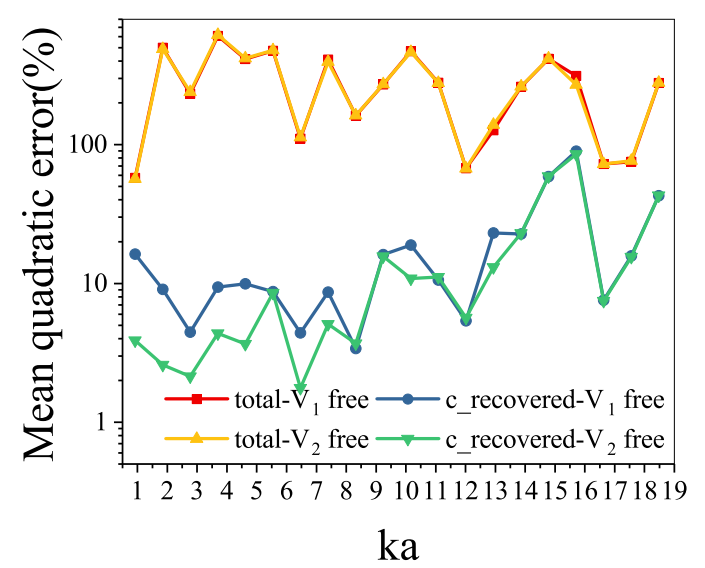

Figure 6. Mean quadratic errors on the measurement surface.

It is clear that the $V_{1}$ free sound field and the $V_{2}$ free sound field are the same in the air because of the weak fluid-structure interaction.

\subsection{In a Bounded Noisy Underwater Environment}

The underwater numerical model is the same as that in the air, but the fluid medium becomes water. The fluid density is $1000 \mathrm{~kg} / \mathrm{m}^{3}$, and the sound speed is $1500 \mathrm{~m} / \mathrm{s}$. The reference sound pressure and reference sound power are $1 \times 10^{-6} \mathrm{~Pa}$ and $1 \times 10^{-18} \mathrm{~W}$, respectively. When the cubic shell vibrates in the water, the characteristic quantity $\lambda$ is greater than 8 in the studied frequency range. Thus, the coupling between the cubic shell and the water is very strong. Considering that the sound pressure radiated by the same vibrating structure in the water is much greater than that radiated in the air with the same vibrational velocity, the strength of the monopole is reduced to $-0.002 i \mathrm{~m}^{3} / \mathrm{s}$ at $100 \mathrm{~Hz}$, which is more reasonable. The position of the monopole is unchanged, and the infinite rigid plane can be simply taken as the bottom of the sea.

The power estimator levels directly obtained and recovered are shown in Figure 7. As mentioned above, the power estimator level in the total sound field is very different from in the $V_{1}$ free sound field. The differences are more than $14 \mathrm{~dB}$ at most frequencies. The power estimator level in the conventional recovered sound field is significantly different from that in the $V_{1}$ free sound field because the interaction between the vibrating structure and the water cannot be ignored. However, the power estimator levels in the conventional recovered sound field separated by the conventional double-layer method and in the $V_{2}$ free sound field are almost the same. The power estimator level in the new recovered sound field recovered by the proposed method agrees well with that in the $V_{1}$ free sound field.

Figure 8 shows the contour map of sound pressure in the $V_{1}$ free sound field, the total sound field, the conventional recovered sound field, the $V_{2}$ free sound field, and the new recovered sound field on $S$. There are obvious differences between the sound pressure distributions of the $V_{1}$ free sound field, the total sound field, and the conventional recovered sound field. However, the sound pressure distributions of the conventional recovered sound field and the $V_{2}$ free sound field are almost identical. The sound pressure distributions of the new recovered sound field and the $V_{1}$ free sound field share the same distributions. 


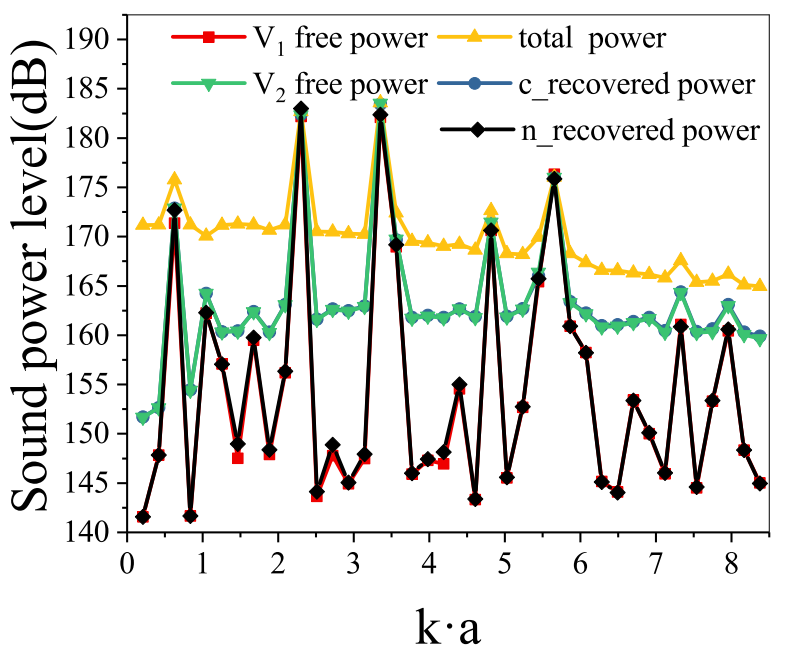

Figure 7. Sound power level on the measurement surface.

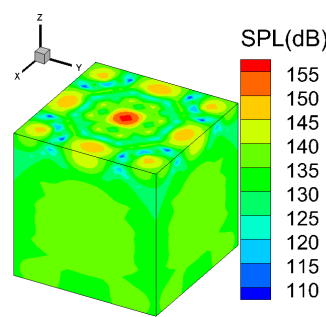

(a) $V_{1}$ free sound field

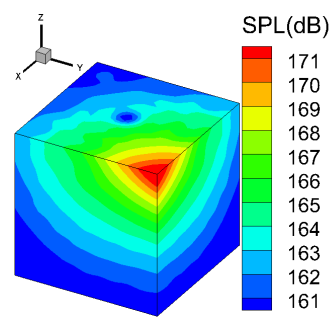

(b) total sound field

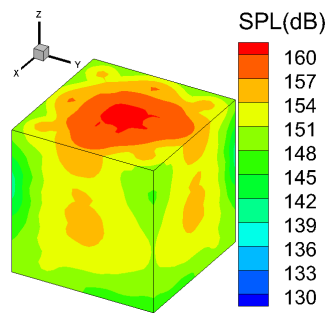

(c) c_recovered sound field

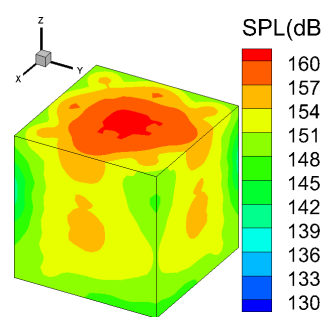

(d) $V_{2}$ free sound field

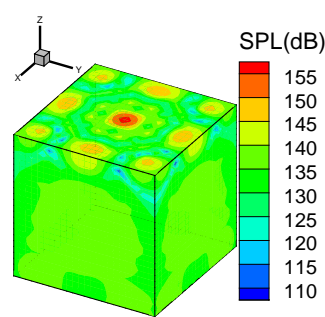

(e) n_recovered sound field

Figure 8. Contour map of sound pressure level (SPL) on the measurement surface at $700 \mathrm{~Hz}(\mathrm{ka}=2.9)$.

Figure 9 shows the mean quadratic errors of the conventional recovered sound field, the new recovered sound field, and the total sound field to the $V_{1}$ free sound field and the $V_{2}$ free sound field. The mean quadratic errors of the total sound field to the $V_{1}$ free sound field and the $V_{2}$ free sound field are both over $100 \%$. Influenced by the strong fluid-structure interaction, the conventional recovered sound field to the $V_{1}$ free sound field is also over $100 \%$. However, the mean quadratic errors of the conventional recovered sound field to the $V_{2}$ free sound field are mostly less than $10 \%$. Additionally, the mean quadratic errors of the new recovered sound field to the $V_{1}$ free sound field are mostly less than $40 \%$. 


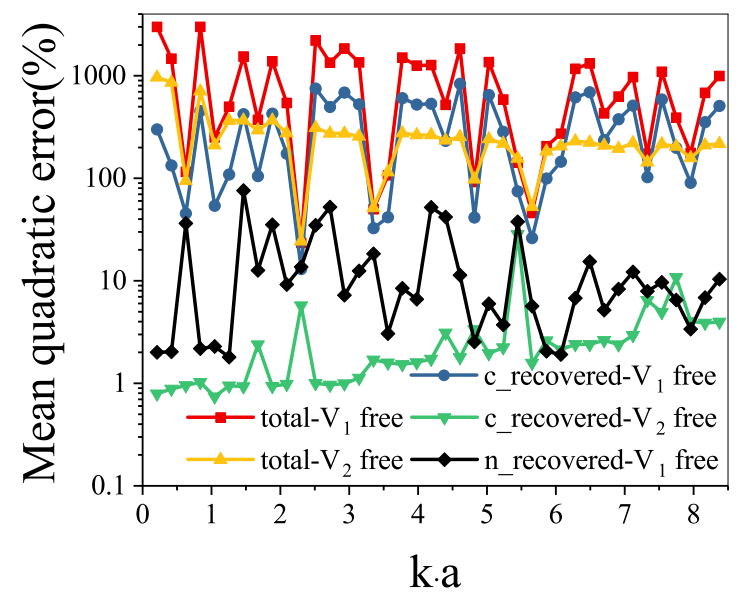

Figure 9. Mean quadratic errors on the measurement surface.

In order to evaluate the robustness of the presented method, the signal-to-noise ratio (SNR) on the $S$ is defined as [22]

$$
S N R=20 \log _{10} \frac{\left\|P^{f}\right\|_{2}}{\left\|P^{t}\right\|_{2}}
$$

where $\|\cdot\|$ represents the 2-norm of a vector; $P^{f}$ and $P^{t}$ represent the free-field and total sound pressure on $S$, respectively.

The relative error is defined as [22]

$$
\text { Error }=\frac{\left\|P^{r e c}-P^{f}\right\|_{2}}{\left\|P^{f}\right\|_{2}}
$$

where $P^{r e c}$ represents the sound pressure recovered by the proposed method on the $S$.

The relative errors at $k a=0.2, k a=4.0$, and $k a=8.4$ when the strengths of the monopole are $-0.0008 i \mathrm{~m}^{3} / \mathrm{s},-0.0032 i \mathrm{~m}^{3} / \mathrm{s},-0.0064 i \mathrm{~m}^{3} / \mathrm{s},-0.0096 i \mathrm{~m}^{3} / \mathrm{s},-0.0128 i \mathrm{~m}^{3} / \mathrm{s}$, and $-0.016 i \mathrm{~m}^{3} / \mathrm{s}$ at $100 \mathrm{~Hz}$ are shown in Figure 10. It can be seen from Figure 10 that the relative error decays with increasing SNR and increases with increasing $\mathrm{ka}$. However, the relative error is always less than $11 \%$. This means that the acoustic characteristics radiated by the structure in the free field could be accurately recovered by the proposed method, even for the SNR up to $-29.5 \mathrm{~dB}$.

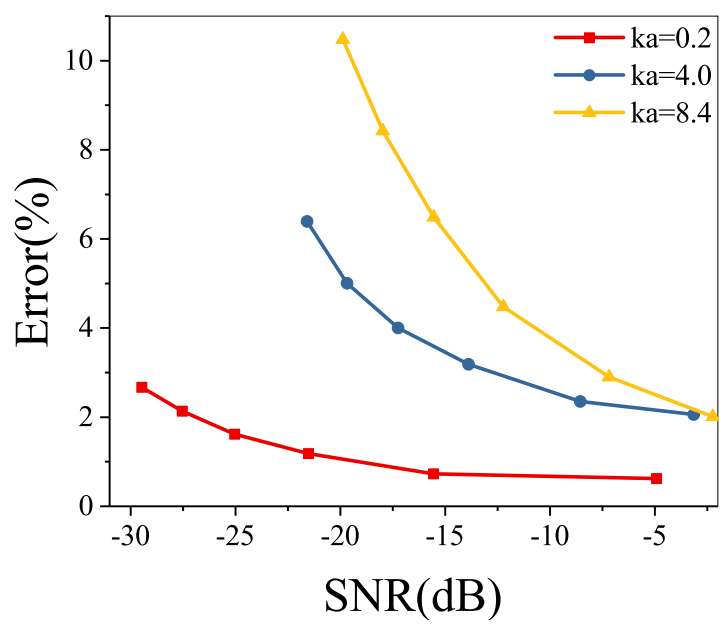

Figure 10. The relative errors of the recovered sound pressure for different frequencies and the SNR. 
In conclusion, the acquisition of the free-field acoustic characteristics of a vibrating structure is more susceptible to the boundaries and the sources of disturbances in underwater environments. Although the strength of the source of disturbance in the water is nearly ten times smaller than that in the air, the source of disturbance has a more significant influence on the vibration of the structure in underwater environments because the interaction between the vibrating structure and the water cannot be ignored. This means that the $V_{1}$ free sound field and the $V_{2}$ free sound field are quite different. The conventional double-layer BEM can only be used to recover the $V_{2}$ free sound field. However, the proposed method can be used to recover the $V_{1}$ free sound field in a bounded noisy underwater environment.

\title{
4. Conclusions
}

The double-layer BEM can only be used to eliminate the incoming sound field and the rigid body scattered sound field. This means that the sound field recovered by this method is the superposition of the free sound field and the elastically radiated sound field. The free-field acoustic characteristics of a structure can be recovered by the double-layer BEM only when the elastically radiated sound field can be ignored such as in the air However, the elastically radiated sound field cannot be ignored in the water because of the strong fluid-structure interaction. To recover the free-field acoustic characteristics of a vibrating structure in a bounded noisy underwater environment, a method combining the BEM with the vibro-acoustic coupling method was presented. Numerical results show that the acoustic characteristics recovered by the presented method in the bounded noisy underwater environment are the same as those radiated by the underwater vibrating structure in the free field. Meanwhile, the proposed method has good robustness and can be used to accurately recover the free-field acoustic characteristics even for the negative SNR. The realization of this method can break through the limitations of the measurement environment to obtain the free-field acoustic characteristics of underwater vibrating structures. However, it should be pointed out that the presented method only can be used with known structural properties. To remedy this, the method [23] based on pressure measurements for constructing the structural impedance should be introduced into the proposed method. It will be studied to reduce the amount of calculation in the future [31-33].

Author Contributions: W.L.: conceptualization, methodology, investigation, software, and writingoriginal draft; S.L.: conceptualization, methodology, resources, supervision, writing-review and editing, project administration, and funding acquisition. All authors have read and agreed to the published version of the manuscript.

Funding: The authors gratefully acknowledge the support of the National Natural Science Foundation of China (grant Nos. 11772080 and 11635004).

Institutional Review Board Statement: Not applicable.

Informed Consent Statement: Not applicable.

Data Availability Statement: Not applicable.

Conflicts of Interest: The authors declared that they have no conflicts of interest related to this work. We declare that we do not have any commercial or associative interest that represents a conflict of interest in connection with the work submitted.

\author{
Abbreviations \\ The following abbreviations are used in this manuscript: \\ FFR Free field recovery \\ BEM Boundary element method \\ NAH Nearfield acoustic holography \\ SONAH Statistically optimized nearfield acoustic holography \\ iPTF inverse patch transfer functions method \\ SIRE Supersonic intensity in reverberant environments
}


ESM Equivalent source method

SPL Sound pressure level

SNR Signal-to-noise ratio

\section{References}

1. Pavić, G.; Du, L. Coupling of sound spaces by plane surface harmonics and its application to sound source characterization. J. Sound Vib. 2019, 440,1-22. [CrossRef]

2. Barnard, A.R.; Hambric, S.A.; Maynard, J.D. Underwater measurement of narrowband sound power and directivity using Supersonic Intensity in Reverberant Environments. J. Sound Vib. 2012, 331, 3931-3944. [CrossRef]

3. Pachner, J. Investigation of Scalar Wave Fields by Means of Instantaneous Directivity Patterns. J. Acoust. Soc. Am. 1956, $28,90-92$. [CrossRef]

4. Weinreich, G.; Arnold, E.B. Method for measuring acoustic radiation fields. J. Acoust. Soc. Am. 1980, 68, 404-411. [CrossRef]

5. Tsukernikov, I.E. Calculation of the field of a sound source in a bounded space. Sov. Phys. Acoust. Ussr 1989, 35, $304-306$.

6. Williams, E.G.; Maynard, J.D.; Skudrzyk, E. Sound source reconstructions using a microphone array. J. Acoust. Soc. Am. 1980, 68, 340-344. [CrossRef]

7. Maynard, J.D.; Williams, E.G.; Lee, Y. Nearfield acoustic holography: I. Theory of generalized holography and the development of NAH. J. Acoust. Soc. Am. 1985, 78, 1395-1413. [CrossRef]

8. Veronesi, W.A.; Maynard, J.D. Nearfield acoustic holography (NAH) II. Holographic reconstruction algorithms and computer implementation. J. Acoust. Soc. Am. 1987, 440, 1307-1322. [CrossRef]

9. Hald, J. Patch holography in cabin environments using a two-layer handheld array with an extended SONAH algorithm. In Proceedings of the Euronoise, Tampere, Finland, 30 May-1 June 2006.

10. Jacobsen, F.; Chen, X.; Jaud, V. A comparison of statistically optimized near field acoustic holography using single layer pressure velocity measurements and using double-layer pressure measurements. J. Acoust. Soc. Am. 2008, 123, 1842-1845. [CrossRef] [PubMed]

11. Aucejo, M.; Totaro, N.; Guyader, J.L. Identification of source velocities on 3D structures in non-anechoic environments: Theoretical background and experimental validation of the inverse patch transfer functions method. J. Sound Vib. 2010, 329, 3691-3708. [CrossRef]

12. Forget, S.; Totaro, N.; Guyader, J.L.; Schaeffer, M. Source fields reconstruction with 3D mapping by means of the virtual acoustic volume concept. J. Sound Vib. 2016, 381, 48-64. [CrossRef]

13. Xiang, S.; Jiang, W.; Pan, S. Sound source identification in a noisy environment based on inverse patch transfer functions with evanescent Green. J. Sound Vib. 2015, 359, 68-83. [CrossRef]

14. Xiang, S.; Jiang, W.; Jiang, H.; Gao, J. Reconstructing the normal velocities of acoustic sources in noisy environments using a rigid microphone array. J. Acoust. Soc. Am. 2016, 140, 2082-2090. [CrossRef]

15. Romano, A.J.; Bucaro, J.A.; Houston, B.H.; Williams, E.G. On a novel application of the Helmholtz integral in the development of a virtual sonar. J. Acoust. Soc. Am. 2000, 108, 2823-2828. [CrossRef]

16. Langrenne, C.; Melon, M.; Garcia, A. Boundary element method for the acoustic characterization of a machine in bounded noisy environment. J. Acoust. Soc. Am. 2007, 121, 2750-2757. [CrossRef] [PubMed]

17. Bi, C.X.; Chen, X.Z.; Chen, J. Sound field separation technique based on equivalent source method and its application in nearfield acoustic holography. J. Acoust. Soc. Am. 2008, 123, 1472-1478. [CrossRef] [PubMed]

18. Bi, C.X.; Stuart Bolton, J. An equivalent source technique for recovering the free sound field in a noisy environment. J. Acoust. Soc. Am. 2012, 131, 1260-1270. [CrossRef] [PubMed]

19. Bi, C.X.; Hu, D.Y.; Zhang, Y.B.; Bolton, J.S. Reconstruction of the free-field radiation from a vibrating structure based on measurements in a noisy environment. J. Acoust. Soc. Am. 2013, 134, 2823-2832. [CrossRef] [PubMed]

20. Bi, C.X.; Hu, D.Y.; Zhang, Y.B.; Jing, W.Q. Identification of active sources inside cavities using the equivalent source method-based free-field recovery technique. J. Sound Vib. 2015, 346, 153-164. [CrossRef]

21. Lin, W.; Li, S.; Liu, S.; Gao, H.; Gao, J.; Liu, Y. An improved method for recovering the acoustic characteristics of a target sound source sitting on plane by boundary element method. Appl. Acoust. 2020, 165, 107316. [CrossRef]

22. Wu, H.; Li, D.; Yu, L.; Jiang, W. A boundary element method based near field acoustic holography in noisy environments. J. Acoust. Soc. Am. 2020, 147, 3360-3371. [CrossRef]

23. Sternini, S.; Rakotonarivo, S.T.; Sarkar, J.; Bottero, A.; Kuperman, W.A.; Williams, E.G. Bistatic scattering of an elastic object using the structural admittance and noise-based holographic measurements. J. Acoust. Soc. Am. 2020, 148, 734-747. [CrossRef]

24. Everstine, G.C.; Henderson, F.M. Coupled finite element/boundary element approach for fluid-structure interaction. J. Acoust. Soc. Am. 1990, 87, 888-896. [CrossRef]

25. Wu, T.W. Boundary Element Acoustics: Fundamentals and Computer Codes; Wit Press: Southampton, UK, 2000.

26. Citarella, R.; Landi, M. Acoustic analysis of an exhaust manifold by Indirect Boundary Element Method. Open Mech. Eng. J. 2011, 5, 138-151. [CrossRef]

27. Xu, Z.; Shen, R.; Hua, H. Structural-Acoustic Coupling Problem of an Immersed Shell by FEM/IBEM. J. Vib. Eng. 2002, 15, 363-367. 
28. Tobocman, W. Calculation of acoustic wave scattering by means of the Helmholtz integral equation. I. J. Acoust. Soc. Am. 1984, 76, 1549-1554. [CrossRef]

29. Schenck, H.A. Improved integral formulation for acoustic radiation problems. J. Acoust. Soc. Am. 1968, 44, 41-58. [CrossRef]

30. Atalla, N.; Bernhard, R.J. Source fields reconstruction with 3D mapping by means of the virtual acoustic volume concept. Appl. Acoust. 1994, 43, 271-294. [CrossRef]

31. Arjunan, A.; Wang, C.; Yahiaoui, K.; Mynors, M.; Morgan, T.; Nguyen, B.; English, M. Sound frequency dependent mesh modelling to simulate the acoustic insulation of stud based double-leaf walls. In Proceedings of the 2014 Leuven Conference on Noise and Vibration Engineering (ISMA2014), Leuven, Belgium, 15-17 September 2014.

32. Idelsohn, S.R.; Onate, E.; Del Pin, F.; Calvo, N. Fluid-structure interaction using the particle finite element method. Comput. Methods Appl. Mech. Eng. 2006, 195, 2100-2123. [CrossRef]

33. Giannella, V.; Lombardi, R.; Pisani, M.M.; Federico, L.; Barbarino, M.; Citarella, R. A Novel Optimization Framework to Replicate the Vibro-Acoustics Response of an Aircraft Fuselage. Appl. Sci. 2020, 10, 2473. [CrossRef] 